\title{
A STUDY ON SENSING TEXT AND RETRIEVAL FROM IMAGE \\ ${ }^{1}$ Anshum Sharma, ${ }^{2}$ Sangeeta Chaudhary, ${ }^{3}$ Mr.Ankur Saxena, and ${ }^{4}$ Shashank Awasthi \\ ${ }^{1}$ Student, Department of Computer Science \& Engineering, G L Bajaj \\ Group of Institutions, Mathura, UP, India \\ ${ }^{2,3}$ Department of Computer Science \& Engineering, G L Bajaj \\ Group of Institutions, Mathura, UP, India \\ ${ }^{4}$ Department of Information and Technology, G L Bajaj Institute of \\ Technology and Management, Greater Noida, India \\ anshumansh04@gmail.com, Sangeeta.chaudhary2626@gmail.com, shashankglbitm@gmail.com
}

\begin{abstract}
Image Retrieval is the essential prerequisite of the present life in the present situation. In light of a colossal measure of various sorts of pictures are included the database from various hotspots for recovery of the picture, various types of preparing are required to remove the important highlights from them. The primary commitment of this paper is to give a short wellspring of reference investigation of different picture Retrieval strategies for example License Plate Localization relying upon the procedures they have utilized and a correlation of every method as far as their benefits, faults, computational power, identification achievement rate and exactness which will be useful for the scientists.
\end{abstract}

Keywords- Content-Based Image Retrieval, Image Retrieval and Histogram

\section{INTRODUCTION}

Intelligent Transportation Systems (ITSs) powerfully affect people's lives these days. The principle purpose of Intelligent transit is to have an effect on the transportation skilfulness and eudemonia to boost the profit by the employment of rising advances and is developed of sixteen different types of innovation based mostly frameworks that area unit classified into clever vehicle frameworks and foundation frameworks. Among them, machine-driven registration number plate Recognition (LPR) Systems assume an indispensable half in several in progress applications together with the concomitant, as an example, electronic instalment frameworks (toll instalment and stopping charge instalment), activity checking frameworks, outskirt crossing control frameworks, ID of stolen vehicles, oil station forecourt reconnaissance frameworks, red light infringement frameworks, vehicle following frameworks, speed implementation control frameworks, ticketing vehicles while not the human management, policing, security and consumer recognizable proof frameworks. Tag numbers extraordinarily distinguish a specific vehicle. Every nation has their own particular tag organize which varies by their formation and hues. So there's a necessity for them to create up the registration number plate Recognition framework cheap for the vehicle registration number plate arranges. Although, the Intelligent Transport Systems intensely rely upon the vigorous Mechanized License Plate Recognition Systems. Trimming a registration code physically with a mouse from the information image is an especially easy strategy, yet with regards to do likewise for any constant or programmed framework, trouble may emerge and won't be appropriate for 
such frameworks. Along these lines, a License Plate Recognition (LPR) System comprises of the accompanying different phases and the framework's fundamental square outline.

1) Image Acquisition from a photographic camera.

2) Vehicle plate Localization (LPL: sleuthing and confirming the placement of the License Plates).

3) Character Segmentation (Segmenting the characters from the License Plates).

4) Character Recognition (Recognizing the divided characters from the License Plates).

In picture securing, the vehicle pictures are procured from computerized cameras; since advanced innovation has their points of interest these days and it is a fundamental stage since it identifies with how to gain top-notch vehicle pictures. License Plate Localization (LPL), which contains the 3 middle of the road steps, for example, Pre-processing, Registration code Detection and vehicle plate Verification.

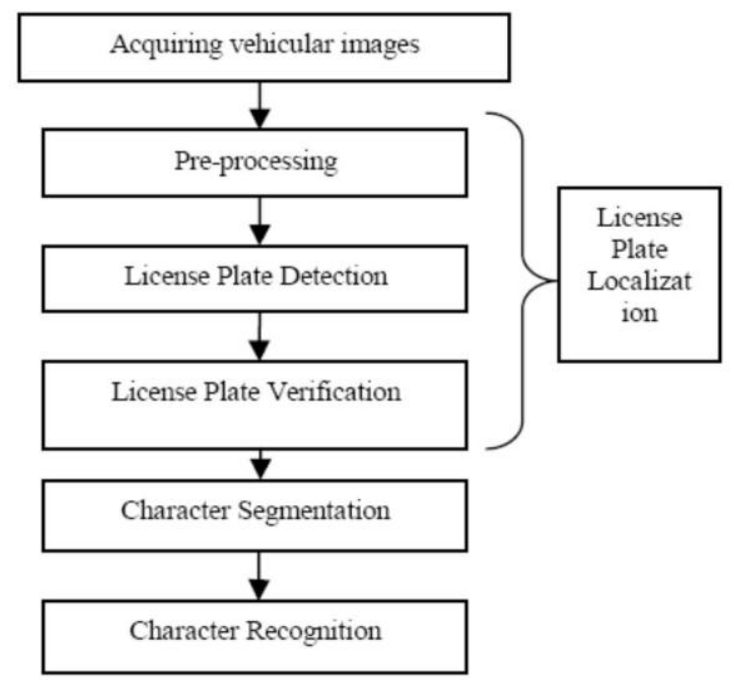

Fig. 1. The basic block diagram of the License Plate Recognition (LPR) system

A few systems have just been created for the proficient License Plate Localization from the given info vehicle pictures lately. A short portrayal of some of the past works is shown during this phase. the standard registration number plate Localization algorithms are essentially classified in to 3 approaches [1] corresponding to color based mostly (based on the colors of the characters within the registration number plate and also the background color), edge-based (based on the sides of the characters and their boundaries) and texture based mostly (based on the pattern specification and unsmooth gift within the License Plates inside the image). The standard techniques for the essential steps for the registration number plate Localization (LPL) square measure mentioned below.

\section{A. Pre-processing}

Pre-processing methods that generally enhance quality of the pictures must get handled, prior to the Vehicle Plate Localization calculation while connected, that builds exactness of the Vehicle Plate Recognition achievement value. Various pre-processing calculations are utilized by the scientists of the vehicle plate Localization, are engaged here during this phase. Initially, the vehicle input photos are modified over into dim photos through the gray scale transformation [2] [3] [4] [5] [6] [7] simply if the calculations for the vehicle plate Localization create utilization of the brilliance knowledge of the image. To remove the noise inside the pictures a nonlinear filter, Median Filtering [2] [3] [8] [12] [16] is employed that replaces the dim estimation of a picture element by the center of the dark estimations of its neighbors. 
Histogram Equalization has been utilized for upgrading the complexity inside the picture, which enhances nature of images along with the consequences of peak-related hustle. Such a significant number of strategies exist for binarization, that is one of the preprocessing method, is utilized to change over gray scale picture into a parallel picture and is used to column the values inside the Vehicle Plates and to smother the foundation points of interest. In Otsu dynamic binarization strategy [9] [10], the picture is divided into sub-districts and to limit associate incentive for every sub-locale is discovered here, that we glance for the sting that limits the intra-class amendment, characterized as a weighted entireness of fluctuations of categories which provides two areas of the image. Edge detection [10] [11] [12] includes the following pre-processing step, which identifies minor differences in picture figure 2 brilliance and in Characteristics of the protest. The vertical fringes made by the License Plate characters are separated by Sobel fringe recognition administrator [5] [12]. Other outline identification administrators are Roberts [6], Canny [6] and Prewitt [13] administrators.
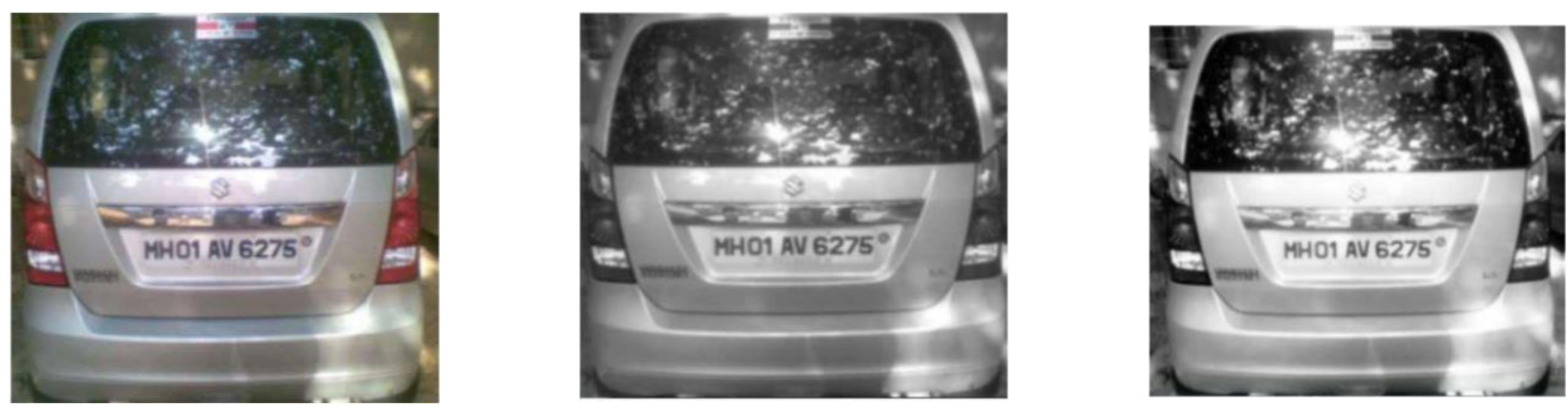

Fig. 2. (a) Loading of image, (b) Gray Scale Conversion, (c) Histogram Equalisation

\section{VEHICLE PLATE LOCALIZATION (DETECTION \& VERIFICATION)}

Satadal Saha, Subhadip Basu, Mita Nasipuri and Dipak Kumar Basu [14] exhibited an Edge-Based Multiorganize technique to the registration number plate Localization from the video previews of noncommissioned vehicles. when Grayscale transformation, the commotions within images are diminished by Median Filtering and therefore the distinction of every picture is improved by bar graph effort. The vertical edges created by the tag characters square measure removed by Sobel edge identification administrator. The featured estimation is sub-categorized to the transitional advances, to be specific recognizable proof of potential band of lines, essential confinement of tag districts in light of measurable dispersion of vertical edge pixels, refinement of tag areas in view of conspicuous vertical edges and restriction of tag bouncing box by evacuating the commotion sections. The accomplishment rate of this calculation was discovered to be $89.2 \%$ and functions laudably for the less uproarious footage since this approach is edge primarily based and provides enticing execution.

\section{METHODOLOGY}

\section{A) Binarization}

The picture is changed over into twofold utilizing adaptive thresholding. In the days of yore, binarization was vital for sending faxes. Nowadays it's as yet essential for things like digitalizing content or division. To begin with, the picture is changed over to grayscale, the edge can either be set settled or versatile utilizing a grouping calculation. 
This algorithm is called Iso Data Algorithm [15]. It first checks the presence of each tone in the picture and tries to locate a decent focus. Binarization is the premise of division.

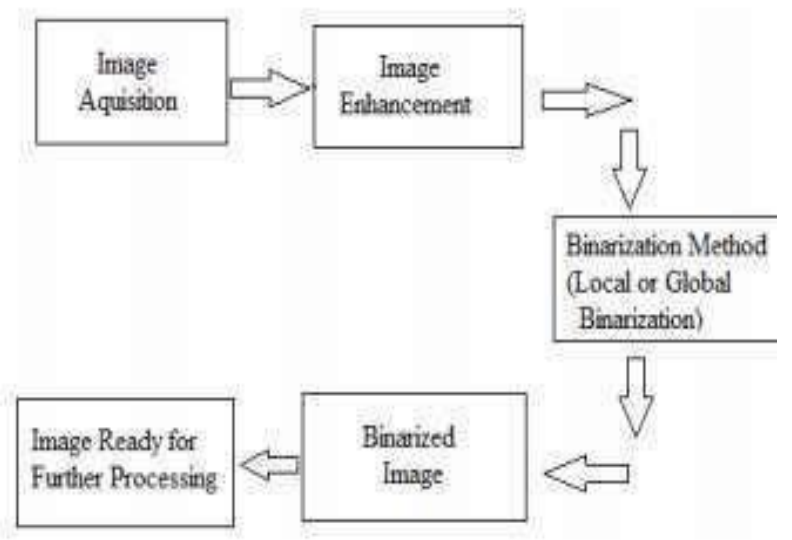

Fig. 3. Block diagram of binarization

The astounding binarized picture can give more exactness in character acknowledgment as an analyzed unique picture since commotion is available in the first picture. Truth be told, issue is what binarization calculation is fitting for all pictures. The choice of most ideal binarization calculation is troublesome in light of the fact that diverse binarization calculation gives an alternate execution on various datasets. This is particularly valid on account of authentic archives pictures with variety conversely and light. The calculations separate into 2 classifications a) global Binarization b) local Binarization. The worldwide binarization methods used single edge an incentive for the complete image and also the near binarization technique wherever the sting esteem computed regionally element by element or district by space.

\section{B) Local Binarization}

\section{1) Adaptive Binarization Method}

\section{Robust bina}

rization strategy [16] has been utilized to perform nearby binarization. Here a window of $\mathrm{NxN}$ squares shove over the whole picture while limit esteem registers for every neighborhood window for binarization. The versatile technique gives more exact outcome when contrasted with Global binarization in such conditions where the picture affected by terrible shading, obscuring, low determination and irregular light. For versatile binarization, one of the processes like Niblack, Sauvola and so on are used to process the neighborhood esteem.

\section{2) Niblack Method}

In Niblack technique [17] the edge an incentive for the neighborhood the window is figured element-wise. The computation of limit esteem relies on the neighborhood mean and standard deviation of window area. The edge esteem is discovering utilizing given condition.

$$
\begin{gathered}
T_{\text {Niblack }}=m+k * s \\
T_{\text {Niblack }}=m+k \sqrt{\frac{1}{N P} \sum\left(p_{i}-m\right)^{2}}
\end{gathered}
$$

Where $\mathrm{m}$, mean of neighborhood of a picture and $\mathrm{s}$, standard deviation of the nearby pixel territory. Here estimation of $\mathrm{k}$ is settled for the value 0.2 (in negative) by the creator. 


\section{3) Sauvola Method}

The Sauvola calculation [18] is an adjusted variety of Niblack calculation. It offers a lot of execution than Niblack underneath such circumstances because the lightweight minor departure from the record image, lightweight surface and then forth. Within the Sauvola modification, the binarization is defined by:

$$
T_{\text {sauvola }}=m *\left(1-k *\left(1-\frac{S}{R}\right)\right)
$$

Where $\mathrm{m}$ comprises of the mean of elements underneath the window zone, $\mathrm{S}$ is the active scope of difference and the estimation of $\mathrm{k}$ limit might be in the scope of values ranging from 0 to 1 . As indicated by [19], the Sauvola technique is superior to Niblack strategy when the dim level pictures change over to the intense and white pictures. The creator settles the $\mathrm{k}$ as 0.5 and $\mathrm{R}$ as 128 .

\section{4) Bernsen method}

Bernsen is neighborhood binarization technique that processes the fringe an incentive from the element of a picture [20]. Here condition used to the count of edge esteem is given underneath:

$$
\begin{aligned}
& T_{\text {Bernsen }}=\left(N_{\text {low }}+N_{\text {high }}\right) / 2 \\
& \text { Where } N_{\text {low }} \text { and } N_{\text {high }} \text { are the grey level values of the } \\
& \text { window. }
\end{aligned}
$$

Where and high are the gray level parameters of the window.

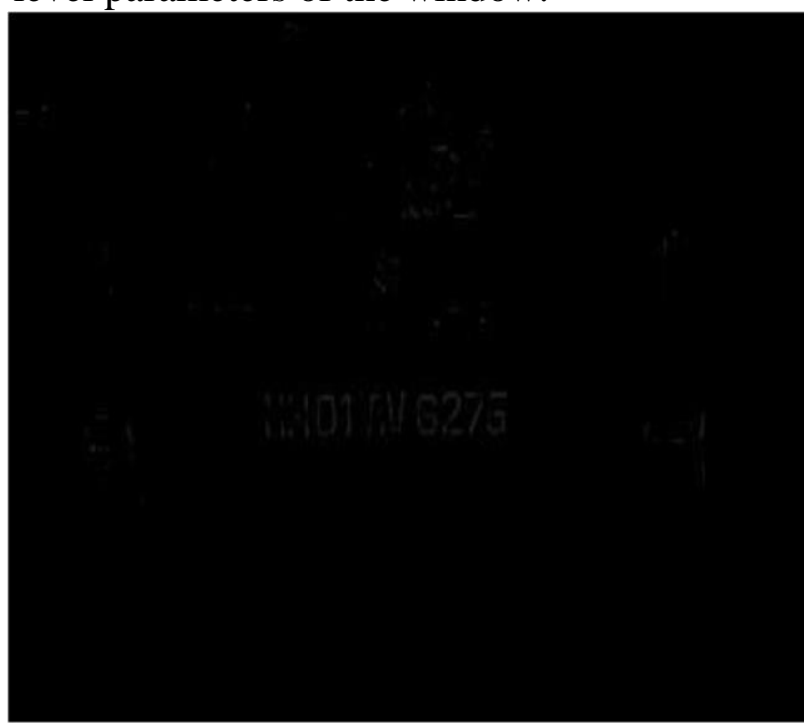

Fig. 4 Count of edge esteem

\section{C) Dilation}

Dilation (normally spoke to by ) is one of the essential activities in numerical morphology. Initially created for twofold pictures, it has been extended first to grayscale pictures, and afterward to finish grids. The expansion activity more often than not utilizes an organizing component for examining and extending the shapes contained in the information picture. Enlargement activity is the switch task of disintegration. While disintegration is for decreasing the frontal area, expansion is for augmenting it. The closer view is extended from its external limit. On the off chance that there is an opening in the closer view, the gap shrivels. Same like disintegration, widening activity is utilizing auxiliary component [21]. The auxiliary component is utilized as a part of convolution with the picture. 


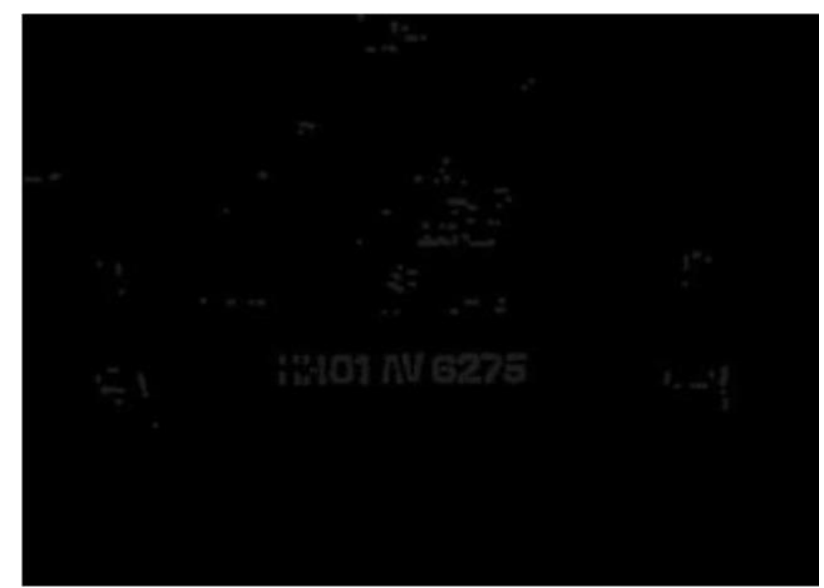

Fig. 5. Auxiliary Component is utilized as a part of convolution with the picture

The expansion procedure will set the foundation pixel to be closer view if there is a piece of the organizing component that achieves the forefront when the focal point of the organizing component still out of sight territory.

\section{D) Edge detection}

The dilated picture is subtracted from the first picture to get the edges. Edge identification assimilates an assortment of scientific ways which account for recognizing particularities in an advanced picture at which the picture brilliance changes forcefully, more formally, has disruptions. The particularities at which picture gleam changes strongly are normally categorized into an arrangement of curved line sections named edges. A known problem of finding disruptions in one-dimensional signs is called as step recognition and the problem of determining signal disruptions after some time is known as change area. Edge discovery is an imperative apparatus in picture preparing, machine view, and PC vision, especially in the territories of feature location and feature extraction. The process of edge detection

1) Leveling: diminishes clamor, without influencing the genuine edges.

2) Enhancement: perform a channel to upgrade the nature of the fringes in the picture (honing).

3) Detection: figure out which edge element ought to be disposed of as clamor while that ought to be held (generally, thresholding gives the basis used to location).

4) Localization: decide the correct area in the edge (sub-pixel determination may be neccessary for a few procedures, i.e., assess the area of a fringe to superior to the dispersing through pixels). Edge shriveling along with connecting is generally needed in this progression.

E) Criteria for ideal edge recognition

(1) Good recognition: the ideal finder must limit the likelihood of false positives (identifying fake edges caused by clamor), and additionally that of false negatives (missing genuine edges).

(2) Good confinement: the edges identified must be as close as conceivable to the genuine edges.

\section{F) Plate region extraction and Character segmentation}

Plate area is discovered by ignoring a rectangular picture the past. Characters are fragmented from the number plate, picture which is then utilized for layout-coordinating as shown in Fig. 6. 


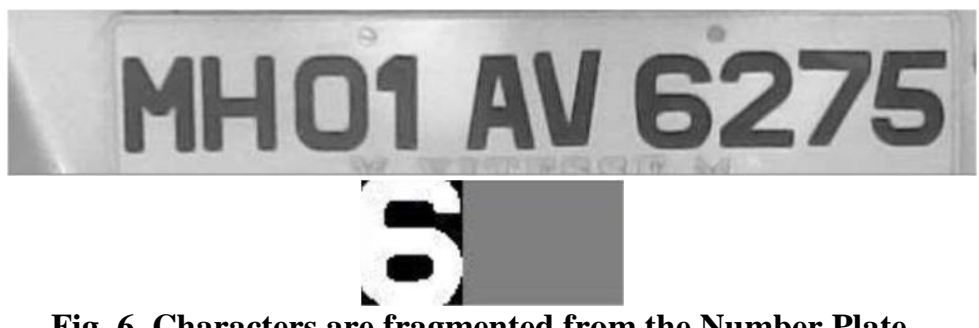

Fig. 6. Characters are fragmented from the Number Plate

\section{CONCLUSION}

Our project is able to successfully detect numbers from some format of number plates. It then stores those numbers in a text file in the first come first serve order. It runs in the following steps. First an image is loaded, then gray scale conversion if performed, then histogram equalization is applied, then Binarization is done on that processed image, then we perform dilation followed by edge detection then plate region extraction and character segmentation is performed. We have made for few formats because for larger format, its data set becomes large and it has to find a match in the bigger range which eventually makes the process slower. Our project finds result with very good accuracy and process very fast. The system works with one limitation that is it reads only simple and straight fonts written on number plate.

\section{REFERENCES}

[1] W. Jia, S. He, and Massimo Piccardi. "Automatic license plate recognition: a review." International Conference Imaging Science, Systems and Technology. CSREA Press, 2004.

[2] Phalgun Pandya and Mandeep Singh, "Morphology Based Approach To Recognize Number Plates in India", International Journal of Soft Computing and Engineering (IJSCE), Vol. 1, No. 3, pp. 107-112, 2011.

[3] Chu-Duc Nguyen, Mohsen Ardabilian and Liming Chen, "Realtime license plate localization based on a new scale and rotation invariant texture descriptor", In proceedings of 11th International conference on Intelligent transportation system, Beijing, pp. 956- 961, 2008.

[4] Muhammad H Dashtban, Zahra Dashtban and Hassan Bevrani, "A Novel Approach for Vehicle License Plate Localization and Recognition”, International Journal of Computer Applications, Vol. 26, No. 11, pp. 22-28, 2011.

[5] Satadal Saha, Subhadip Basu and Mita Nasipuri, "Automatic Localization and Recognition of License Plate Characters for Indian Vehicles", International Journal on Comp Science and Engineering Technology, Vol. 2 No. 4, pp. 520-534, 2011

[6] Anish Lazrus, Siddhartha Choube and Sinha, "An Efficient Method of Vehicle Number Plate Detection and Recognition", International Journal of Machine Intelligence, Vol. 3, No. 3, pp. 134-137, 2011

[7] Baoming Shan, "Vehicle License Plate Recognition Based on Text-line Construction and Multilevel RBF Neural Network", Journal of Computers, Vol. 6, No. 2, pp. 246-254, 2011.

[8] Pawan Wawage and Shraddha Oza, "Design Approach for Vehicle License Plate Automatic Detection and Character Recognition System Using Classification Algorithm", International Journal of Emerging Technology and Advanced Engineering, Vol. 2, No. 10, pp. 132-135, 2011.

[9] Vahid Abolghasemi and Alireza Ahmadyfard, “An edge-based color-aided method for license plate detection", Image and Vision Computing, Vol. 27, No. 8, pp. 1134-1142, 2009. Muhammad H Dashtban, Zahra Dashtban, Hassan Bevrani, A Novel Approach for Vehicle License Plate Localization and Recognition, International Journal of Computer Applications (0975 - 8887), Volume 26- No.11, July 2011.

[10] Yo-Ping Huanga, Chien-Hung Chenb, Yueh-Tsun Chang and Frode Eika Sandnes, "An intelligent strategy for checking the annual inspection status of motorcycles based on license plate recognition", Expert Systems with Applications, Vol. 36, No. 5, pp. 9260-9267, 2009.

[11] Satadal Saha, Subhadip Basu, Mita Nasipuri and Dipak Kumar Basu, "License Plate Localization from Vehicle Images,An Edge Based Multi-stage Approach”, International Journal of Recent Trends in Engineering, Vol. 1, No. 1, pp. 284-289, 2009.

[12] Binarization, http://felixniklas.com/imageprocessing/binarization. 


\section{Gyancity Journal of Electronics and Computer Science \\ Vol.3, No.2, pp. 20-27, September 2018 \\ ISSN:24462918 DOI: 10.21058/gjecs.2018.32003}

[13] Derek Bradley, Gerhard Roth, "Adaptive Thresholding Using the Integral Image”.

[14] Khurram Khurshid, Imran Siddiqi, Claudie Faure, Nicole Vincent, "Comparison of Niblack inspired Binarization methods for ancient documents", 16th International conference on Document Recognition And Retrieval, 2009.

[15] J. Sauvola, T. Seppänen, S. Haapakoski, M. Pietikänen, “Adaptive document binarization” , Fourth International Conference Document Analysis and Recognition (ICDAR), p. 147-152, Ulm, Germany, August 1997.

[16] K. Khurshid, I. Siddiqi, C. Faure, N. Vincent, "Comparison of Niblack inspired Binarization methods for ancient documents", 16th International conference on Document Recognition and Retrieval, USA, 2009.

[17] Madhuri Latha, Chakravarthy, "An Improved Bernsen Algorithm Approaches For License Plate Recognition", IOSR Journal of Electronics and Communication Engineering (IOSR-JECE) ISSN: 2278-2834, ISBN: 2278- 8735. Volume 3, Issue 4 (SepOct. 2012) 